\title{
EFFECTIVENESS OF MATRIX RHYTHM THERAPY FOR FROZEN SHOULDER IN BREAST CANCER RELATED LYMPHEDEMA PATIENT: A CASE REPORT
}

\section{T.V.Gnanasekar.}

Physiotherapist, Grace Physiotherapy and Rehab Center, India.

\section{ABSTRACT}

Background. Frozen shoulder, also known as adhesive capsulitis limits shoulder range of motion (ROM)among Breast Cancer Related Lymphedema (BCRL) patients. Although several therapeutic interventions exist to treat BCRL symptoms, no consensus has been reached thus far.

Case Report:This case study examined the use of Matrix Rhythm Therapy (MRT)for Frozen Shoulder in BCRL patient along with Complete Decongestive Therapy (CDT).The patient presented with chief complaints of pain and restricted shoulder movements secondary to mastectomy.MRT was administered for 60-75 minutes in 10 sessions over a period of three weeks.

Results.Notable changes were observed post-intervention in ROM (from 100 to 180 degrees), pain (from 7 to 2), quality of life (QOL) (from 53 to 17 ) and limb volume (from $43.54 \%$ to $27.35 \%$ ).

Conclusion.MRT was found to be beneficial in this case in a short period of time, however, large-scale studies are required to affirm the impact of $M R T$ as it is cost effective.

KEYWORDS: Secondary BCRL symptoms, Matrix Rhythm Therapy (MRT), Complete Decongestive Therapy (CDT), Frozen Shoulder, Mastectomy, Vibration Therapy.

Address for correspondence: Dr. T.V. Gnanasekar (CLT, OR),Physiotherapist,Grace Physiotherapy and Rehab Center, India. E-Mail: gnanasekar@gracephysio.in

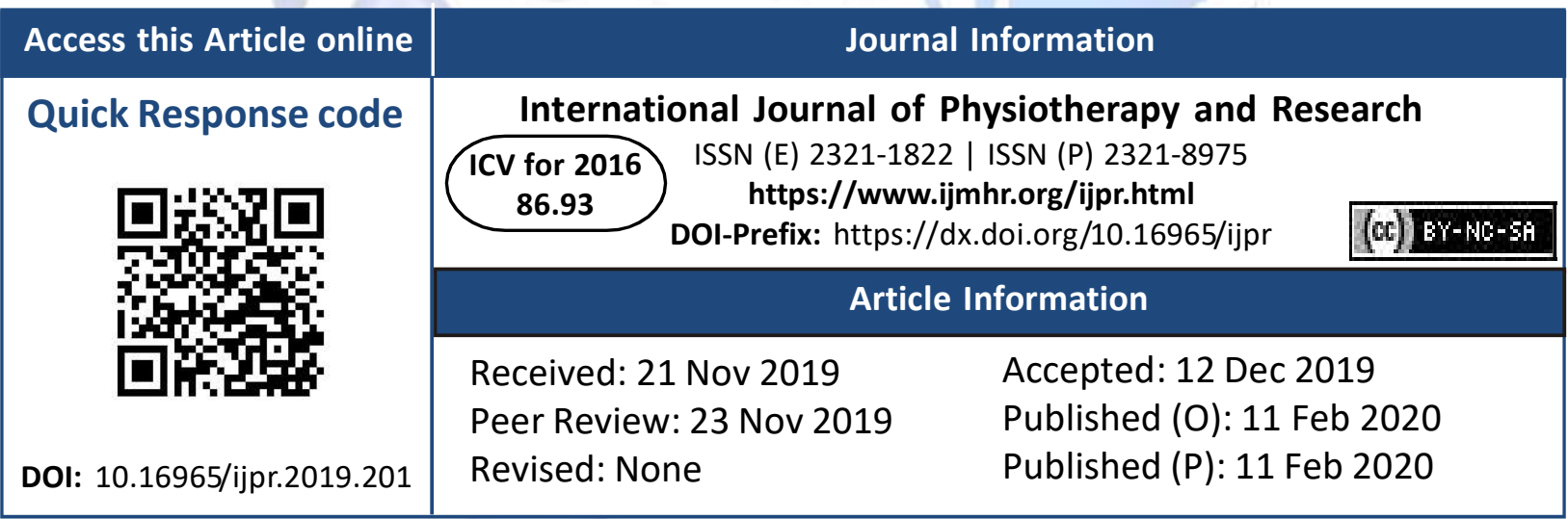

\section{INTRODUCTION}

Breast cancer-related lymphedema (BCRL) is a common problem occurring in 1 out of 5 breast cancer $(\mathrm{BC})$ patients with an incidence of $5 \%$ to $50 \%$. The risk factors of BCRL could be treatment-related or due to the individual patient factors [1].The four components - Manual Lymphatic Drainage (MLD), compression garments, physical activity and skin care comprise the Complete Decongestive Therapy (CDT), which is the mainstay of lymphedema treatment[2]. Apart from pain, swelling, tightness and heaviness of arm, BC survivors also experience secondary complications like reduced shoulder and arm range of motion
(ROM), pain due to augmented fluid tension in subcutaneous tissues, Iymphangio-sarcoma, cellulites, slow recovery of the tissues and risk of other infections[3].

Shoulder pain is a common problem and is considered as a predictor for Quality of Life (QOL) after surgery[4]. BCRL can cause or aggravate shoulder pain because of which patients may experience reduced $\mathrm{QOL}$ and prolonged hospital stay [5]. Reduced ROM was presented in BCsurvivors especially in the first month post-surgery which persisted even after 12 months, irrespective of the treatment modality. Misunderstanding that lymphedema and its 
secondary symptoms are self-recuperative and unawareness of the role of physiotherapy in mitigating these symptoms has been observed in BCRL patients.Timely attention and medical care provided to $B C R L$ is important in terms of recovery and routine activities [6]. Several therapeutic interventions exist to treat impaired ROM of the arm secondary to lymphedema, but no consensus has been reached as to what constitutes optimal or definitive treatment.

Matrix Rhythm Therapy (MRT) is a vibromassage technique working on the concept that all the tissues of the body oscillate on the same frequency as brain's alpha rhythm i.e. 8 to 12 $\mathrm{Hz}$. This is said to be the frequency at which the body maintains homeostasis. Any external injury or trauma can disrupt this frequency and thereby inducing pain and impeding function [7].

The objective of this study was to assess the effectiveness of MRT on frozen shoulder in BCRL patient with respect to $R O M$, pain, limb volume and QOL.

\section{CASE REPORT}

Patient Information: The patient is a 50 -year old woman from Chennai, India. She was diagnosed with carcinoma in the right breast and had undergone Radical Mastectomy on June $6^{\text {th }} 2016$ and completed three cycles of Fluorouracil, Adriamycin and Cytoxan (FAC) chemotherapy. The patient visited the clinic after two years after surgery on November $24^{\text {th }}, 2018$. Her presenting symptoms were pain in the right shoulder along with restriction in shoulder movements which was prevalent for the past three months. She was unsuccessful with her attempts of self-care and alternative treatment methods involving hot fomentation.Post-operation, the patient had fever but recovered at the time of discharge. The patient had a history of diabetes mellitus and was on medication. During physical examination, residue in the upper outer quadrant, skin puckering, with axillary lymphadenopathy and restriction in range of motionof right shoulder were observed.

The purpose of the study was explained to patient and a written informed consent was obtained.This case report is presented based on the consensus-based clinical case reporting guideline[8].
Clinical Findings and Diagnostic Assessment: $X$ ray of the right shoulder was taken to confirm adhesive capsulitis. Pain intensity score was 7 as measured by a ten point Verbal Rating Scale (VRS). Shoulder ROM was assessed to be $100 u$ u using goniometer. Arm circumferences were measured at two points using a flexible, non-stretch, woven fabric tape (in centimeter). Measurements were taken on both arms with the first point on the knuckle of Metacarpophalangeal joint (MCP), the second at the line of wrist followed by a difference of $4 \mathrm{cms}$ each. During the measurements, the participant was standing, with her arms at her sides and elbows straight. The compression sleeves had been removed an hour before measurements were taken and limb volume was calculated using Limb Volume Program (LVP).LVP is a software developed by the Academy of Lymphatic Studies, to help therapists monitor the patient performance by calculating the difference in limb volume of the affected and unaffected extremity from the volume (in millilitres) of fluid. The recorded pre-testscore was 43.5\%. QOL was assessed using Lymphedema Life Impact Scale (LLIS) which was found to be a score of 53 . LLIS is a comprehensive tool specific to lymphedema condition, consisting of 18 items to evaluate the impediment caused in physical, psychosocial, and functional domains due to the condition[9].

Physiotherapy Intervention: The patient lied down at ease and powder was applied by the therapist in areas requiring MRT (around deltoid, pectorals, trapezes, scapula pectorals and axilla) to prevent friction induced by the probe. MRT was administered by longitudinal stroking and pushing the probe onto soft tissues including shoulder joint line, axilla and scapula.

The patient's intervention can be said as having begun the day she visited the clinic (24-11-2018). With each intervention session lasting 60-75 minutes (inclusive of CDT), the patient attended 10 sessions separated by a one-day break between each session, spread over a period of 3 weeks. In all sessions, MRT was followed by CDT in which the patient received MLD, gleno-humeral mobilization and deep breathing exercises. MLD strokes were applied to the oedematous limb, starting at the 
base of neck and progressing to the affected limb. MLD was directed proximally from upper arm to axilla, and then from hand to elbow. Finally, the whole limb was drained from the distal to the proximal portion and low elastic short stretch Multi-Layered Compression bandages were applied.

The patient was regular to the sessions, could tolerate the treatment and followed instructions for self-care at home which included shoulder mobilization exercises, self-MLD and self-bandaging.Muscle soreness was observed in patient after the sessions but was bearable. The patient found the treatment affordable and did not find it financially straining.

\section{RESULTS}

The pre- and post-test values of primary outcomes showed remarkable difference. At the end of the 10-session physiotherapy intervention, ROM improved $180^{\circ}$ from the initial $100^{\circ}$. Difference in pre- and post-intervention scores in abduction, adduction, internal and external rotation indicated improvement in ROM (Fig. 1). Pain score on the VRS had dropped considerably to 2 from the initial score of 7.The limb volume decreased from $43.54 \%$ to $27.35 \%$.The post intervention LLIS score of 17 (pre-test score- 53) indicates that the patient's QOL increased markedly because lower the score, better the QOL.The patient responded well to therapy with reduced pain, improvedLLIS score, reduced limb volume and significantly improved range of motion in the shoulder.

Fig. 1:Shoulder ROM before and after three weeks of MRT in BCRL patient with frozen shoulder.

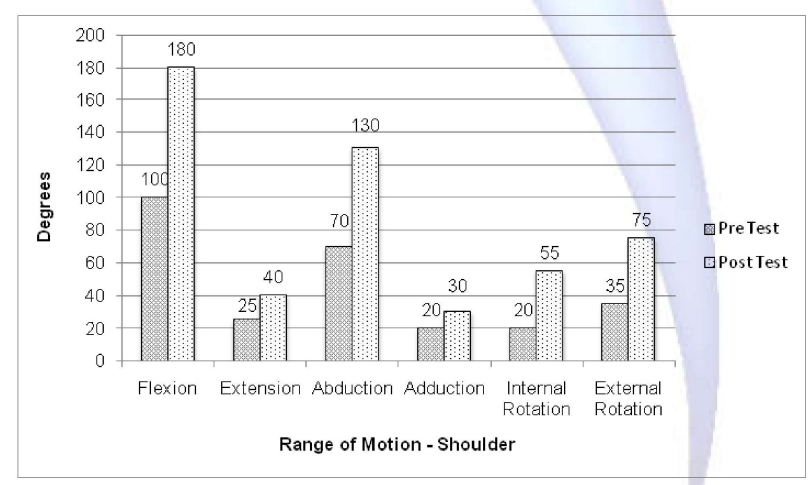

\section{DISCUSSION}

The current study is one of the few to examine the effect of MRT on BCRL symptoms. The study showed substantial improvement with regards to ROM, pain, limb volume and QOL post-intervention.

MRT is a relatively less explored model in physiotherapy intervention. MRT helps in improving active and passive ROM, sensory function and muscle strength [7].

MRT performed in lymphedema of the limb showed no significant difference in limb volume after 20 sessions given 5 days per week. This might be because the study did not employ CDT in the patient[10]. MRT significantly improves blood flow in areas of its application [11] and increased lymph accumulation might result in production of lymph in edematous tissues or from extrinsic pumping due to vasomotion $[12,13]$. Therefore, it is absolutely necessary to provide CDT to patients undergoing MRT, which was performed in our study. In the current study, we observed muscle soreness in the patient after MRT sessions which might be due to the duration of MRT given; the effects were comparable to a study conducted on frozen shoulder [14].

There were only two previous studies conducted on lymphedema patients, both of which did not mention the exact duration for which MRT was administered $[10,15]$. Naik et al. revealed improved ROM of frozen shoulder and reduction in pain by $30 \%$ in VAS just after one sitting of MRT [14]. Future studies need to explore the optimum duration of MRT intervention.

Many interventions are available for treating secondary BCRL symptoms including yoga, aqua training and resistance and aerobic exercise[16,17]. Any of these methods entail progressive physical activity and would require at least three-four weeks to exhibit its efficacy. However, our study participant was able to show improved shoulder function over three weeks without the need to perform physically straining activities. BCRL women with impaired ROM felt physically restricted as it affected their daily activities and also had to forego leisure activities [18].

In our study, there was a 36-point difference in LLIS score indicating that the patient must have had significantchange in her QOL[9].

Reduction in Hospital Anxiety and Depression scale (HAD) was demonstrated by a study 


\section{post-MRT session [15].}

There were a few limitations in our study. Possibility of degenerative rotator cuff pathology occurring in patients regardless of BCRL was not ruled out in this study. The duration of lymphedema in our patient was defined as the duration since first self-report of symptoms and there was uncertainty as to when the exact diagnosis using objective measures was done.

\section{CONCLUSION}

MRT along with a CDT was helpful in improving range of motion of shoulder, pain, arm volume and QOL in breast cancer related lymphedema condition. The results indicate that MRT in combination with CDT could be effective in treating frozen shoulder in BCRL patients. Further large scale RCT studies should be conducted to confirm the findings of this case study.

\section{ABBREVIATIONS}

ROM - Range of Motion

BCRL - Breast Cancer-Related Lymphedema

MRT -Matrix Rhythm Therapy

CDT -Complete Decongestive Therapy

QOL - Quality of Life

BC - Breast Cancer

MLD - Manual Lymphatic Drainage

FAC-Fluorouracil, Adriamycin and Cytoxan

VRS - Verbal Rating Scale

MCP - Metacarpophalangeal

LVP - Limb Volume Program

LLIS - Lymphedema Life Impact Scale

HAD -Hospital Anxiety and Depression

\section{ACKNOWLEDGEMENTS}

This manuscript was supported by Dr.Vidhubala, Dr.Niraimathi and Ms. Malini. Their valuable inputs and moderation helped direct this paper.

\section{Conflicts of interest: None}

\section{REFERENCES}

[1] Gillespie TC, Sayegh HE, Brunelle CL, Daniell KM, Taghian AG. Breast cancer-related lymphedema: Risk factors, precautionary measures, and treatments. Gland Surg 2018;7:379-403. doi:10.21037/ gs.2017.11.04.
[2] Jeffs E, Ream E, Taylor C, Bick D. Clinical effectiveness of decongestive treatments on excess arm volume and patient-centered outcomes in women with early breast cancer-related arm lymphedema. JBI Database Syst Rev Implement Reports 2018;16:453506. doi:10.11124/JBISRIR-2016-003185.

[3] Herrera JE, Stubblefield MD. Rotator cuff tendonitis in lymphedema: a retrospective case series. Arch Phys Med Rehabil 2004;85:1939-42. doi:10.1016/ j.apmr.2004.06.065.

[4] Jang D-H, Kim M-W, Oh S-J, Kim JM. The Influence of Arm Swelling Duration on Shoulder Pathology in Breast Cancer Patients with Lymphedema. PLoS One 2015;10:e0142950. doi:10.1371/journal.pone. 0142950.

[5] Nesvold I-L, Dahl AA, Løkkevik E, Marit Mengshoel A, Fosså SD. Arm and Shoulder Morbidity in Breast Cancer Patients after Breast-conserving Therapy versus Mastectomy. Acta Oncol (Madr) 2008;47:835-42. doi:10.1080/0284186080 1961257.

[6] Hidding JT, Beurskens CHG, Van Der Wees PJ, Van Laarhoven HWM, Nijhuis-van Der Sanden MWG. Treatment related impairments in arm and shoulder in patients with breast cancer: A systematic review. PLoS One 2014;9. doi:10.1371/ journal.pone.0096748.

[7] Randoll U. Matrix-Rhythm-Therapy: Utilizing the entrainment effect to optimize body performance and tissue regeneration in chronic diseases. Centre-Vedantique-GeneveOrg n.d.

[8] Gagnier JJ, Kienle G, Altman DG, Moher D, Sox H, Riley D, et al. Author's personal copy The CARE guidelines: consensus-based clinical case report guideline development. J Clin Epidemiol 2014;67:46-51. doi:10.1016/j.jclinepi.2013.08.003.

[9] Weiss J, Daniel T. Validation of the Lymphedema Life Impact Scale (LLIS): A Condition-specific Measurement Tool for Persons with Lymphedema. Lymphology 2015;48:128-38.

[10] Narin AN, Alpozgen AZ, Kulli HD. Effects of matrix rhythm therapy on primary lymphedema: A case report. J Phys Ther Sci 2016;28:2418-21. doi:10.1589/jpts.28.2418.

[11] Taspinar F, Aslan UB, Sabir N, Cavlak U. Implementation of matrix rhythm therapy and conventional massage in young females and comparison of their acute effects on circulation. J Altern Complement Med 2013;19:826-32. doi:10.1089/acm.2012.0932.

[12] Moore JE, Bertram CD. Lymphatic System Flows. Annu Rev Fluid Mech 2018;50:459-82. doi:10.1146/ annurev-fluid-122316-045259.

[13] Ikomi F, Kawai Y, Ohhashi T. Recent Advance in Lymph Dynamic Analysis in Lymphatics and Lymph Nodes. Ann Vasc Dis 2012;5:258-68. doi:10.3400/ avd.ra.12.00046.

[14] Naik V, Bhagwat S, Pathania T, Bootwala F. Effectiveness of Matrix Rhythm Therapy in Frozen Shoulder with respect to ROM and Pain - An Experimental Study. Int J Appl Res 2018;4:73-6. doi:2394-5869. 
[15] Bhatikar K. Evaluation of Long-Term Effect of Clinical Physiotherapy Treatment Protocol for Post Cancer Mastectomy Induced Lymphadenopathy and Restricted Joint Range: A Novel Protocol Case Study. J Integr Oncol 2018;07. doi:10.4172/23296771.1000219.

[16] Li L, Yuan L, Chen X, Wang Q, Tian J, Yang K, et al. Current Treatments for Breast Cancer-Related Lymphoedema: A Systematic Review. Asian Pac J Cancer Prev 2016;17:4875-83. doi:10.22034/ APJCP.2016.17.11.4875.

[17] Baumann FT, Reike A, Reimer V, Schumann M, Hallek $M$, Taaffe DR, et al. Effects of physical exercise on breast cancer-related secondary lymphedema: a systematic review. Breast Cancer Res Treat 2018;170:1-13. doi:10.1007/s10549-018-4725-y.
[18] Voogd AC, Ververs JMMA, Vingerhoets AJJM, Roumen $\mathrm{RMH}$, Coebergh JWW, Crommelin MA. Lymphoedema and reduced shoulder function as indicators of quality of life after axillary lymph node dissection for invasive breast cancer. Br J Surg 2003;90:76-81. doi:10.1002/bjs.4010.

How to cite this article: T.V.Gnanasekar. EFFECTIVENESS OF MATRIX RHYTHM THERAPY FOR FROZEN SHOULDER IN BREAST CANCER RELATED LYMPHEDEMA PATIENT: A CASE REPORT. Int J Physiother Res 2020;8(1):3354-3358. DOI: 10.16965/ijpr.2019.201 